\title{
MODELACIÓN Y SIMULACIÓN BASADA EN AGENTES COMO ALTERNATIVA PARA EL ESTUDIO DE LAS ORGANIZACIONES EMPRESARIALES
}

\author{
Karol D. Cepeda-Susatama ${ }^{1}$, Kelly A. Durango-Ruiz ${ }^{2}$, Luz E. Bohórquez-Arévalo ${ }^{3}$ \\ ${ }^{1}$ Estudiante de Ingeniería Industrial, Universidad Distrital Francisco José de Caldas, Bogotá, Colombia. Correo \\ electrónico: kdcepedas@correo.udistrital.edu.co \\ ${ }^{2}$ Estudiante de Ingeniería Industrial, Universidad Distrital Francisco José de Caldas, Bogotá, Colombia \\ ${ }^{3}$ Doctor en Ciencias de la Dirección, profesor asociado, Universidad Distrital Francisco José de Caldas, Bogotá, \\ Colombia
}

Fecha de recibido: 10 de octubre del $2016 \quad$ Fecha de aprobado: 20 de marzo del 2017

Cómo citar este artículo: K. Cepeda-Susatama, K. Durango-Ruiz, L. Bohórquez- Arévalo, "Modelación y simulación en agentes como alternativa para el estudio de las organizaciones empresariales", Ingeniería Solidaria, vol. 13, n. ${ }^{\circ} 22$, mayo de 2017, pp. 103-119. doi: http://dx.doi.org/10.16925/in.v13i22.1838

Resumen. Introducción: el artículo es producto de la investigación "Modelación y simulación basada en agentes como alternativa para el estudio de las organizaciones empresariales", desarrollada durante el 2016 en la Universidad Distrital Francisco José de Caldas, en Bogotá. Los autores sintetizan la literatura más sobresaliente publicada sobre la modelación y simulación de sistemas complejos a través de la historia, en particular de los modelos basados en agentes, con el fin de desarrollar una apreciación de sus posibles contribuciones a la investigación en administración. Metodología: se analiza la información recolectada por medio de los artículos científicos obtenidos de las bases de datos ProQuest y Scopus desde 1990 hasta la fecha, con la inclusión de conceptos claves de modelación y simulación, basadas en agentes descritos en algunos libros. Resultados: los enfoques tradicionales, caracterizados por el uso de la estadística y la matemática clásica no son pertinentes para modelar organizaciones por su visión determinista y reduccionista, por lo cual se presenta un enfoque alternativo para el estudio de las organizaciones que se encuentra en las técnicas de modelación y simulación desde las ciencias de la complejidad, cuyo fin es estudiar sistemas caracterizados por comportamientos emergentes. Conclusiones: a pesar de su baja popularidad, la implementación de estas alternativas no solo puede beneficiar a las organizaciones, sino que puede aportar en la construcción de lineamientos para una mayor apropiación de modelos de simulación para organizaciones.

Palabras claves: modelación, organizaciones empresariales, sistemas complejos, sistemas sociales. 


\title{
Agent-based Modeling and Simulation as an Alternative for the Study of Business Organizations
}

\begin{abstract}
Introduction: The article arises from the research Agent-based modeling and simulation as an alternative for the study of business organizations, conducted during 2016 at the Universidad Distrital Francisco José de Caldas, Bogotá. The authors summarize the most outstanding literature published on the modeling and simulation of complex systems throughout history, particularly agent-based models, in order to form an opinion on their possible contributions to management research. Method: We analyzed the information collected from scientific articles in ProQuest and Scopus databases from 1990 to date, including key concepts of modeling and simulation, based on agents described in some books. Results: Traditional approaches, characterized by the use of statistics and classical mathematics, are not relevant to model organizations because of their deterministic and reductionist view. Therefore, an alternative approach is presented for the study of organizations that are found in the modeling and simulation techniques of the sciences of complexity, whose purpose is to study systems characterized by emerging behaviors. Conclusions: Despite its low popularity, the implementation of these alternatives cannot only benefit organizations, but can also contribute to the creation of guidelines for greater appropriation of simulation models for organizations.
\end{abstract}

Keywords: modeling, business organizations, complex systems, social systems.

\section{MODELAÇÃO E SIMULAÇÃo BASEADAS EM AGENTES COMO ALTERNATIVA PARA O ESTUdO DAS ORGANIZAÇÕES EMPRESARIAIS}

Resumo. Introdução: este artigo é produto da pesquisa "Modelação e simulação baseadas em agentes como alternativa para o estudo das organizações empresariais", desenvolvido em 2016 na Universidad Distrital Francisco José de Caldas, em Bogotá. Os autores sintetizam a literatura mais destacada publicada sobre modelação e simulação de sistemas complexos através da história, em particular dos modelos baseados em agentes, a fim de desenvolver uma apreciação de suas possíveis contribuições para a pesquisa em administração. Metodologia: analisa-se a informação coletada por meio dos artigos científicos obtidos das bases de dados ProQuest e Scopus desde 1990 até os dias atuais, com a inclusão de conceitos-chave de modelação e simulação baseadas em agentes descritos em alguns livros. Resultados: as abordagens tradicionais, caracterizadas pelo uso da estatística e da matemática clássica, não são pertinentes para modelar organizações por sua visão determinista e reducionista; portanto, apresenta-se uma abordagem alternativa para o estudo das organizações que se encontra nas técnicas de modelação e simulação desde as ciências da complexidade, cujo fim é estudar sistemas caracterizados por comportamentos emergentes. Conclusões: apesar de sua baixa popularidade, a implantação dessas alternativas não somente pode beneficiar as organizações, mas sim pode contribuir para a construção de lineamentos para uma maior apropriação de modelos de simulação para organizações.

Palavras-chaves: modelação, organizações empresariais, sistemas complexos, sistemas sociais. 


\section{Introducción}

Las organizaciones empresariales $y$, de manera amplia, el entorno en que actúan, son sistemas complejos caracterizados por la emergencia permanente de nuevas situaciones a partir de la interacción entre los agentes que los componen [1]. Son sistemas altamente sensibles a la incertidumbre, y sus comportamientos no corresponden a la intensión de los individuos que los generan, lo que produce inesperados y contados resultados intuitivos [2].

La modelación y simulación de sistemas complejos (MSSC) es una poderosa herramienta para el avance de la investigación en sistemas sociales y, en particular, en organizaciones empresariales, principalmente por la capacidad de representar sistemas con altos niveles de complejidad y facilitar la toma de decisiones. Sin embargo, su adopción para la investigación en disciplinas como la administración y, específicamente, para la investigación en ciencias sociales, ha sido lenta por el poco conocimiento que los investigadores en esos campos tienen respecto a los métodos y técnicas aplicadas para la modelación y simulación, así como sus bondades y limitaciones [3].

Por esta razón, se ha optado por el estudio de las organizaciones por medio de los enfoques tradicionales, principalmente la dinámica de sistemas. El pensamiento newtoniano que caracteriza los enfoques tradicionales para la modelación, se torna inadecuado si se reconoce que, tanto las organizaciones empresariales, como las problemáticas a las que se enfrenta, tienen muchas variables y/o agentes en interacción que cambian rápidamente (modificando su relevancia). Además, los enfoques tradicionales se caracterizan por identificar las variables relevantes en el comportamiento del sistema, asumiendo la posibilidad de aislar el objeto de estudio de otros sistemas con los que interactúa debido a que no ejercen un impacto notorio, así como de reducir su complejidad a variables clave.

El principio de causalidad que caracteriza los enfoques tradicionales para la construcción de modelos es un rasgo propio de la mecánica clásica o física de Newton, según la cual es posible deducir la evolución futura del sistema a partir del conocimiento de las condiciones iniciales. Los sistemas causales expresan un enfoque reduccionista y determinista. Son reduccionistas, en el sentido de querer asignar causas a las diferentes situaciones que se presentan en la realidad; y son deterministas, en la medida en que buscan proyectar el surgimiento de fenómenos o el logro de ciertos estados a partir de las causas identificadas y los parámetros definidos.

Los modelos soportados en la estadística y la matemática clásica presentan fuertes limitantes cuando se aplican al estudio de sistemas que intercambian información con el entorno y que se encuentran caracterizados por altos números de agentes en interacción, como claramente son los sistemas sociales humanos [4].

Un enfoque alternativo para la modelación y simulación de sistemas sociales humanos -y en particular de organizaciones empresariales- que permita superar las restricciones de los enfoques tradicionales, se encuentra en las ciencias de la complejidad. Estas ciencias se ocupan del estudio de sistemas caracterizados por rupturas en las que los estados de orden se rompen, dando lugar a nuevas situaciones diferentes a las condiciones iniciales. La termodinámica del no equilibrio, la ciencia de redes, la teoría del caos, la teoría de catástrofes, la ciencia de fractales o las lógicas no clásicas, entre otras, se enmarcan dentro de las ciencias de la complejidad [5].

La modelación y simulación de sistemas complejos desde las ciencias de la complejidad se ocupa del estudio de la emergencia de propiedades novedosas a partir de la interacción entre amplios números de agentes. En las organizaciones empresariales, la MSsc facilita la comprensión, por ejemplo, del surgimiento de patrones culturales [6], de la actuación conjunta entre dos o más personas [7], de las formas de organización empresarial que permiten mayores niveles de adaptación [8], de la formulación de políticas empresariales [9], de la influencia de la turbulencia y complejidad del ambiente en el diseño organizacional [10], entre otros fenómenos complejos en los que no es posible ni correcto identificar las variables relevantes, dado el alto nivel de aspectos en interacción.

La MSsc, desde las ciencias de la complejidad, se soporta en una variedad de investigaciones previas como la filosofía del organismo [11], redes neuronales [12], la cibernética [13], y la teoría general de sistemas [14], entre otras. Los mayores avances en MSsc se encuentran en la matemática y la computación. Su aplicación en la investigación en administración ha sido lenta y dispersa geográficamente, aunque desde el 2002 se evidencian mayores esfuerzos al respecto [15]. 
Es importante señalar que las ciencias de la complejidad se ocupan de problemas relacionados pero distintos a los abordados por la cibernética organizacional (se encuentran relacionados porque estudian sistemas complejos no lineales) [16]. Sin embargo, la cibernética organizacional se ha enfocado en sistemas sociales humanos, y su mayor aporte se encuentra en el modelo de sistema viable desarrollado por Beer $[17,18]$, el cual se orienta a la búsqueda de la viabilidad de la organización. De manera amplia, se puede afirmar que el principal aporte que las ciencias de la complejidad le hacen a la investigación en sistemas complejos - como claramente lo son las organizaciones empresariales y el entorno en el que actúan-, se encuentra en las técnicas para la modelación y simulación.

La modelación y la simulación, desde esta perspectiva de pensamiento, se conocen como sociedades artificiales o modelación basada en agentes [19]. La modelación y simulación basada en agentes (MSBA) ha sido la técnica de mayor aplicación para la investigación en ciencias sociales, y en particular en el estudio de las organizaciones empresariales [11, 20]. Este tipo de simulación se caracteriza por la existencia de muchos agentes que interactúan usualmente en ausencia de un controlador central, en el que los comportamientos emergentes que se presentan son difíciles de predecir y/o anticipar debido a la capacidad adaptativa entre las partes.

\section{Metodología implementada}

Para la elaboración del presente artículo se consultaron diferentes bases de datos bibliográficas, tales como ProQuest, Scopus e IEeE Xplore Digital Library, entre otras. Se realizó una búsqueda inicial enfocada en la dinámica de sistemas y su aporte al estudio de la complejidad en las organizaciones empresariales a nivel global. De los artículos de interés encontrados se establecieron como relevantes aquellos que se fundamentaban en el estudio de la ingeniería, lo cual generó aportes importantes al presente estudio.

La segunda búsqueda se estableció al tomar en cuenta la modelación y simulación basada en agentes y su influencia en el estudio de la administración de las organizaciones empresariales complejas. A partir de dichas fuentes se revisaron los estudios y trabajos a fin de obtener la información y lograr hacer la comparación expuesta en la sección de resultados.

Mediante el análisis de los artículos enfocados en la programación y simulación de sistemas complejos, para el interés del presente estudio, en las organizaciones empresariales se identificaron los principales aportes, tanto de la dinámica de sistemas, como de la modelación basada en agentes, con el propósito de conocer sus ventajas y desventajas, además de obtener las herramientas adecuadas en el estudio de la complejidad en este tipo de organizaciones, y establecer la mejor técnica para comprender el comportamiento de sistemas cambiantes.

\section{La complejidad creciente de las organizaciones empresariales y del entorno en el que actúan}

Los sistemas complejos se caracterizan por la emergencia permanente de nuevos comportamientos (nuevos estados de orden) a partir de la interacción entre los agentes [21]. Las organizaciones empresariales y el entorno en el que actúan son sistemas complejos, y más exactamente son sistemas de complejidad creciente, debido a la ganancia y generación de nueva información que surge como resultado de los altos niveles de interdependencia con otros sistemas con los que interactúan (sistemas económicos, políticos, culturales, religiosos, etc.) [22]. La expresión "complejidad creciente" se utiliza para denotar que no todos los sistemas aumentan su complejidad; de hecho, es posible encontrar sistemas que tienden a reducirla o conservarla.

La globalización y el desarrollo de las tecnologías de la información han incrementado la interdependencia de los sistemas sociales humanos (sistemas económicos, políticos, culturales, tecnológicos, etc.), y están promoviendo la creación de redes que facilitan el surgimiento y propagación de innumerables situaciones difíciles de predecir y controlar [23]. Estas situaciones influyen en el comportamiento de las organizaciones empresariales de maneras insospechadas. El intercambio de productos, los flujos de información, las crisis financieras, las crisis ambientales, el crimen organizado, la migración global, los conflictos geopolíticos, las enfermedades infecciosas, las guerras civiles y el terrorismo, entre otros fenómenos, 
generan situaciones que se propagan rápidamente e influyen en el desempeño empresarial.

La complejidad creciente de las organizaciones empresariales y del entorno en el que actúan se visualiza en las situaciones de incertidumbre política, social, económica, ambiental, cultural, religiosa, empresarial, etc. Esto permite comprender a las organizaciones como sistemas alejados del equilibrio, es decir, sistemas caracterizados por la presencia de altas tasas de cambio. Los sistemas alejados del equilibrio no pueden ser explicados a partir del comportamiento de variables clave o de variables de mayor impacto; son sistemas no causales en los que pequeñas causas pueden desencadenar grandes, pequeños o medianos efectos; no son equivalentes a sistemas caóticos, pues la interdependencia permite la emergencia permanente de nuevos estados de orden, y en general, la interacción entre las partes hace que el sistema se vea como un todo organizado $[24,25]$.

La complejidad creciente pone de manifiesto la imposibilidad de estudiar las organizaciones empresariales como sistemas estáticos, sistemas aislados, o a partir de variables claves, por lo que surge la necesidad de encontrar nuevas alternativas que faciliten el estudio de las organizaciones como sistemas alejados del equilibrio y caracterizados por la emergencia de comportamientos difíciles de predecir y/o anticipar.

\section{Resultados}

\subsection{La modelación y simulación de sistemas complejos}

La modelación y simulación son herramientas complementarias. Los modelos permiten representar el sistema, y dependiendo del lenguaje utilizado pueden ser matemáticos, computacionales, lógicos, conceptuales, o teóricos. La simulación es un tipo especial de modelo que permite experimentar artificialmente sobre el sistema, a fin de explorar diferentes comportamientos y alternativas de solución. La simulación no hace referencia exclusiva a la programación en computador [5], sino a las vías para el desarrollo de experimentos mentales que, a diferencia de los experimentos reales, se soportan en información de un mundo simulado, lo cual implica la construcción de una realidad virtual [26].
La modelación y simulación proporcionan una perspectiva diferente en la investigación del problema, y no se constituyen en un fin en sí mismo. Algunas de las ventajas de la modelación y simulación son: (a) claridad y precisión para comunicar las ideas; (b) facilita la comprensión del sistema a estudiar; (c) permite experimentar con nuevas situaciones; (d) facilita la validación de supuestos; (e) genera menos costos que si se utiliza en el mundo real; (f) menores restricciones en el tamaño de la muestra; $(\mathrm{g})$ mayor capacidad para rastrear con precisión las condiciones que generan determinados comportamientos emergentes; y (h) facilidad para monitorear comportamientos emergentes entre agentes diversos, entre otras [27, 28].

La modelación y simulación de sistemas complejos permite ampliar el conocimiento de sistemas que, en razón a su tamaño o complejidad, no es posible observar de manera completa o de experimentar con ellos. El universo, los mercados y las organizaciones empresariales son algunos ejemplos de sistemas que se pueden observar de manera muy pobre, y la experimentación puede implicar altos costos económicos y/o sociales. También, permite comprender complejas interacciones entre múltiples procesos interdependientes, en los que los resultados no se pueden predecir o anticipar.

La modelación y simulación de sistemas complejos ha sido considerada como una tercera vía para hacer ciencia [29], es decir, se presenta como un método paralelo a los dos que han marcado el progreso científico: (a) el análisis teórico o deducción; y (b) el análisis empírico o inducción. La MSsc empieza con un conjunto de supuestos explícitos, pero, a diferencia de la deducción, no prueba teoremas. Los datos en la MssC pueden ser analizados inductivamente, en oposición a la inducción típica, pues no se comienza a partir de un conjunto de reglas rigurosamente especificadas. Mientras que el propósito de la inducción es encontrar patrones, y el de la deducción, las consecuencias de un conjunto de supuestos, el propósito de la MSsc es ayudar a la intuición [19].

Esta tercera vía para hacer ciencia no desconoce los éxitos que, tanto la deducción, como la inducción, han generado en el progreso científico. Sin embargo, la dificultad de solución matemática que caracteriza a los sistemas conformados por grandes números de agentes con patrones de interacción cambiantes y adaptativos, ha estimulado el uso de la Mssc como la alternativa para facilitar la comprensión de dichos sistemas [30]. Este 
problema parece ser común en las ciencias sociales y ha llevado a los investigadores a elegir supuestos, como, por ejemplo, la racionalidad individual, a fin de facilitar la modelación [31].

Por lo tanto, la MSsC facilita la exploración de todas las alternativas posibles. Estos modelos "ponen a jugar al sistema" de tal forma que las distintas jugadas pueden llegar a diversas soluciones. Desde esta perspectiva, y a diferencia de los enfoques tradicionales para la modelación, no se busca identificar una alternativa de solución. La MSsC permite descubrir los comportamientos emergentes a partir de conjuntos simples de supuestos, y en consecuencia, es posible identificar múltiples alternativas de solución. Los agentes racionales son un tipo ideal de agente que se introduce en las ciencias sociales de manera análoga a como se introduce en la física la teoría de los gases ideales o de los fluidos perfectos, entre otras. El conocimiento de tales tipos ideales es útil para facilitar cálculos aproximados. Pero, en la realidad, los gases reales se apartan sistemáticamente de los gases ideales, los fluidos reales tienen viscosidad, y los agentes racionales se dejan influir e influyen de manera no proporcional a otros con los que interactúan (son adaptativos), lo que hace que la racionalidad ofrezca una visión limitada y poco real del comportamiento del agente, y por extensión de la organización [31].

La MSsc ha adquirido amplia relevancia en los últimos años por la facilidad que brinda para el estudio de agentes adaptativos más que racionales [19]. Esta característica, unida a la posibilidad de comprender los comportamientos emergentes que surgen en el sistema a partir de la interacción entre las partes, explica su pertinencia en el estudio de sistemas sociales humanos.

Existen en la literatura diferentes tipologías de modelos de simulación de sistemas complejos con aplicación en sistemas sociales humanos [32, 34]. Los modelos basados en agentes y los autómatas celulares son algunas de las técnicas utilizadas [1]. Los modelos basados en agentes han sido la técnica de mayor aplicación en los últimos años, y de acuerdo con diversos autores [35-37], es la técnica más pertinente para estudiar los comportamientos emergentes en sistemas humanos. En el siguiente apartado se describen las principales características de esta técnica de simulación.

James March se considera pionero en el uso de la simulación computacional en las ciencias sociales $[38,39]$, es decir, no es novedad que investigaciones en ciencias sociales se soporten en modelación y simulación. Sin embargo, su uso en la administración ha tenido un lento nivel de crecimiento en comparación con la aplicación en matemática, las ciencias de la computación, e incluso en economía y psicología [36]. De hecho, durante los años ochenta aparecieron en las principales revistas de administración investigaciones soportadas en simulación [40-43]. En los años noventa se empiezan a encontrar con cierta regularidad, aunque escasas en comparación con otras disciplinas, investigaciones que utilizan métodos de simulación computacional [3]. Desde el 2000, las investigaciones que se soportan en Mssc se comportan en cantidad de manera similar en la administración, la matemática y las ciencias de la computación, y después del 2011 las investigaciones que aplican MSsC crecen a una tasa superior en administración en comparación con la matemática y las ciencias de la computación [15].

El lento crecimiento que la aplicación de MSsC ha tenido en la investigación en administración con respecto a la matemática y las ciencias de la computación, puede ser explicado por la poca importancia que las escuelas de administración y, de manera amplia, de ciencias sociales, le dan al estudio de técnicas para la modelación y simulación de sistemas complejos $[3,15]$. Lo anterior ha llevado a que muchos investigadores no tengan los antecedentes formativos necesarios para elegir las técnicas para modelar y simular el sistema, o para evaluar e interpretar resultados de simulaciones.

\subsection{Modelación basada en agentes}

La modelación y simulación basada en agentes (Agent-Based Modeling and Simulation ABMs) es una técnica de modelado emergente que estudia la dinámica bottom-up, considerada por diversos autores como la manera más adecuada para estudiar sistemas sociales [44-46]. Lo anterior en razón a la capacidad de exhibir patrones emergentes a partir de la interacción entre agentes heterogéneos que aprenden de sus experiencias, y tienen la capacidad de influir, ser influidos y adaptarse a las condiciones cambiantes del entorno, es decir, comprende la interacción de agentes que desarrollan comportamientos adaptativos más que racionales.

A través de la historia, los autores han llamado a este tipo de modelado de diferentes formas: modelación basada en agentes [34, 47-49], modelos multi-agente [50], simulación social basada en agentes 
[51], y modelación y simulación basada en agentes [37, 52-54]. Sin embargo, su significado no difiere, y permite ampliar su campo de estudio.

La ABMs tiene importantes antecedentes en las investigaciones en sistemas complejos [21, 55-56] y vida artificial $[52,57]$. El énfasis en las formas en que los sistemas colectivamente se autoorganizan o se adaptan a las condiciones emergentes del entorno, se constituyen en los rasgos que permiten distinguir la ABMs de otras técnicas de simulación, como la dinámica de sistemas.

Un modelo típico basado en agentes tiene tres elementos [37]: (a) un conjunto de agentes; (b) un conjunto de relaciones y modos de interacción entre los agentes; y (c) el entorno en el que actúan.

Es importante señalar que no existe consenso universal con respecto a la definición de agente, más allá de la propiedad esencial de autonomía. Algunos autores insisten en que un agente es aquel que desarrolla comportamientos adaptativos, que es capaz de aprender y cambiar en respuesta a las experiencias que tenga con el entorno, sin necesidad de la existencia de un controlador central que determine su comportamiento- autonomía [37].

El conjunto de relaciones y los modos de interacción entre agentes hacen referencia a quiénes interactúan con quién, y de qué manera. En la ABMs los agentes interactúan con otros y con el entorno, lo que no significa que todos interactúen con todos. La interacción entre los agentes genera subredes que permiten evidenciar la existencia de agentes mejor conectados que otros (más cantidad de amigos, afiliaciones, etc.), tal como sucede en el mundo real. Las formas de interacción permiten insertar mayores niveles de complejidad en la modelación. La interacción entre los agentes puede ser de colaboración, competencia, interacción directa, interacción indirecta, entre otros. En la tabla 1 se presentan diferentes mecanismos de interacción y sus características. Adicionalmente, no todos los vínculos de interacción entre los agentes tienen el mismo nivel de intensidad. Por ejemplo, la interacción entre dos compañeros de trabajo que además son amigos, y comparten diferentes espacios sociales, es más fuerte que la de dos personas que, si bien trabajan en la misma organización, rara vez intercambian información.

Tabla 1. Enfoques del ABMs según su mecanismo

\begin{tabular}{|l|l|}
\hline \multicolumn{1}{|c|}{ Mecanismos } & \multicolumn{1}{c|}{ Casos en ssH } \\
\hline $\begin{array}{l}\text { Interacciones } \\
\text { directas }\end{array}$ & $\begin{array}{l}\text { El desarrollo de una idea dentro de la organización comienza con una persona que busca la } \\
\text { solución a un problema. Esta idea se va propagando a través de la organización, pero sin salir } \\
\text { de esta, por ser información confidencial de la misma. }\end{array}$ \\
\hline $\begin{array}{l}\text { Estigmergia } \\
\text { colaboración a } \\
\text { través de medio } \\
\text { físico) }\end{array}$ & $\begin{array}{l}\text { Las ideas que se desarrollan en una organización tienen siempre algo que mejorar. En este sen- } \\
\text { tido, el ser humano aprovecha estas vulnerabilidades para comenzar y desarrollar un proyecto } \\
\text { nuevo, de manera autónoma, a partir de diversos planteamientos hechos por otras personas, ya } \\
\text { sea dentro o fuera de la organización. }\end{array}$ \\
\hline Refuerzo & $\begin{array}{l}\text { Los seres humanos tienden a adoptar comportamientos diferentes dependiendo del ambiente } \\
\text { en el que se estén desenvolviendo, teniendo en cuenta las relaciones que necesite para su adap- } \\
\text { tación al mismo. En este sentido, se puede identificar mediante el comportamiento que tiene } \\
\text { una persona en su trabajo y el que tiene en su casa. }\end{array}$ \\
\hline Cooperación & $\begin{array}{l}\text { La organización de los individuos se torna cooperativa cuando dos personas tienen conoci- } \\
\text { mientos diferentes, pero deben trabajar en conjunto para responder oportunamente a los cam- } \\
\text { bios en el ambiente. }\end{array}$ \\
\hline $\begin{array}{l}\text { Arquitectura } \\
\text { genética }\end{array}$ & $\begin{array}{l}\text { Esta se determina bajo la filosofía de los holones* sociales, considerando al ser humano como } \\
\text { un holón por su integralidad y por ser parte de uno mayor llamado "sociedad", en la que se } \\
\text { evidencian los comportamientos individuales y colectivos a la vez. }\end{array}$ \\
\hline
\end{tabular}

* Holón es algo que es un todo y una parte a la vez. Puede ser desde una partícula subatómica hasta un planeta.

Fuente: modificación de [58] 
En la ABMs los agentes actúan en información local (no todos los agentes están conectados con todos), y en ausencia de controlador central que determine el comportamiento del sistema. La interacción de los agentes con el entorno les permite influir, ser influidos, y modificarse en respuesta a las condiciones cambiantes del medio en el que se encuentran.

La ABMs es una técnica de amplio interés para la investigación en diversas disciplinas, y brinda diferentes posibilidades, de acuerdo con el uso que se le asigne. En las ciencias sociales, los modelos de agentes se han empleado para estudiar el comportamiento de los mercados de valores [59], comprender la dinámica en las decisiones de compra del consumidor [60], los comportamientos dinámicos en las cadenas de suministro [61], la evolución en las decisiones de inversión y reinversión de propietarios de vivienda en sus barrios [62], evaluar la eficacia de las estrategias desarrolladas [63], y evaluar la rapidez y efectividad de estrategias de difusión de información [64], entre otros.

Sin embargo, al realizar la revisión de grupos y líneas de investigación en modelación y simulación de sistemas complejos en el listado de las mejores cien escuelas de negocios y/o administración del mundo según Forbes (2015), tan solo la Escuela de Negocios dela Universidad de Northwestern Kellogg Business School, y la Escuela de Administración de la UCLA Anderson, trabajan hoy en día la temática.
En las seis principales revistas de administración del mundo según scimago Journal \& Country Rank, se han encontrado en los últimos seis años investigaciones que se soportan en la ABMs para estudiar la interacción dinámica entre la acción y la memoria en las rutinas organizativas [65], validar el enfoque de los costos de transacción en la creación de empresas [66], evaluar diferentes alternativas de gobierno en función de las relaciones de alianza entre organizaciones [67], y para la formulación de políticas que propendan a la igualdad estructural en las formas de gobierno de las corporaciones [9], tal como se observa en la figura 1.

De acuerdo con la revisión realizada de los "journals of management" mejor clasificados en el escalafón se observa que menos del $1 \%$ de las producciones escritas publicadas se relacionan con el tema del ABMS, es decir, en promedio, menos de un artículo anual, demostrando el bajo interés por la investigación en este tema desde la perspectiva de la gestión organizacional.

Como se observa en la tabla 2, los artículos publicados del 2010 al 2016 se enfocan en el aspecto social de la organización y sus comportamientos, al igual que la gobernabilidad y el aspecto económico, concluyéndose que el estudio de los comportamientos sociales en las organizaciones y las actuaciones conjuntas, siendo un tema de gran interés y utilidad, no ha sido aprovechado para el uso del management.

Artículos totales $\quad$ Artículos sobre ABMS

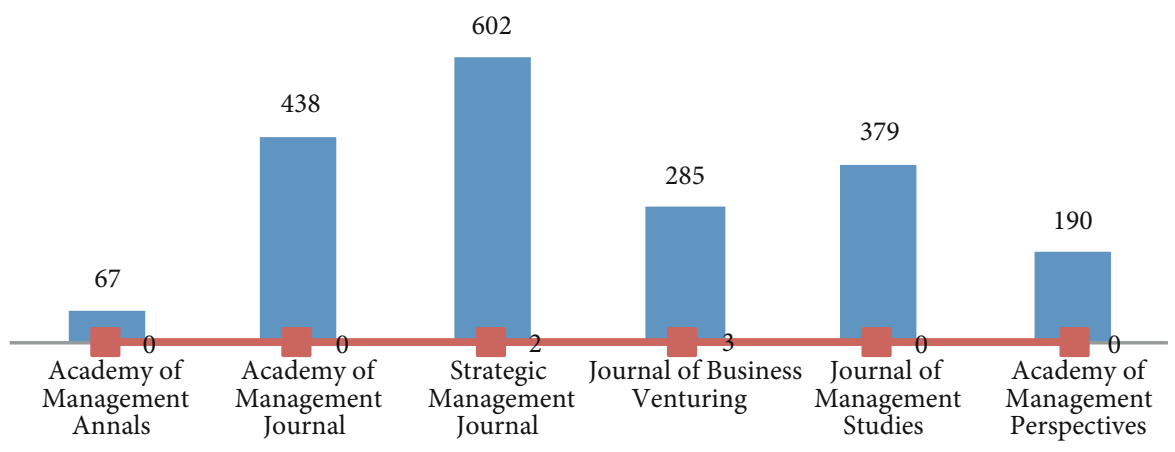

Figura 1. Artículos relacionados con management y ABMs en scImago Journal \& Country Rank, en el área de "Business, Management and Accounting" y en la categoría "Business and International Management"

Fuente: elaboración propia 
Tabla 2. Artículos relacionados con ABMs en el área de management en el periodo 2010-2016

\begin{tabular}{|c|c|c|c|}
\hline Journal & Fecha & Autores & Título \\
\hline \multirow{2}{*}{$\begin{array}{l}\text { Strategic } \\
\text { Management } \\
\text { Journal }\end{array}$} & Julio del 2011 & $\begin{array}{l}\text { Aggarwal, V.A., } \\
\text { Siggelkow, N., } \\
\text { Singh, H. }\end{array}$ & $\begin{array}{l}\text { Governing collaborative activity: Interdependence and the } \\
\text { impact of coordination and exploration }\end{array}$ \\
\hline & Junio del 2014 & $\begin{array}{l}\text { Kogut, B., } \\
\text { Colomer, J., } \\
\text { Belinky, M. }\end{array}$ & $\begin{array}{l}\text { Structural equality at the top of the corporation: Mandated } \\
\text { quotas for women directors }\end{array}$ \\
\hline \multirow{3}{*}{$\begin{array}{l}\text { Journal of } \\
\text { Management } \\
\text { Studies }\end{array}$} & $\begin{array}{l}\text { Diciembre del } \\
2013\end{array}$ & $\begin{array}{l}\text { Mcmullen, J.S. , } \\
\text { Dimov, D. }\end{array}$ & $\begin{array}{l}\text { Time and the entrepreneurial journey: The problems and } \\
\text { promise of studying entrepreneurship as a process }\end{array}$ \\
\hline & Enero del 2015 & Bylund, P.L. & $\begin{array}{l}\text { Signifying Williamson's contribution to the transaction cost } \\
\text { approach: an agent-based simulation of coasean transaction } \\
\text { costs and specialization }\end{array}$ \\
\hline & $\begin{array}{l}\text { Diciembre del } \\
2016\end{array}$ & $\begin{array}{l}\text { Miller, K.D., } \\
\text { Pentland, B.T., } \\
\text { Choi, S. }\end{array}$ & $\begin{array}{l}\text { Dynamics of performing and remembering organizational } \\
\text { routines }\end{array}$ \\
\hline
\end{tabular}

Fuente: elaboración propia

Lo anterior, en contraste con la aparición de revistas especializadas en simulación, y en las que aparecen resultados de investigaciones en campos de interés de la administración que hacen uso de la MSsC (European Journal of Operational Research, Stochastic Processes and their Applications, Mathematical Models and Methods in Applied Sciences, Journal of Nonlinear Science, Communications in Nonlinear Science and Numerical Simulation, y Journal on Applied Dynamical Systems, entre otros).

Como metodología de investigación científica, es posible identificar al menos tres propósitos que puede tener la aplicación de la ABMs: predicción, prueba de hipótesis y explicación [30]. La predicción en la simulación permite develar relaciones entre variables que pueden ser consideradas predicciones de simulación o hipótesis, y que posteriormente pueden ser sometidas a pruebas empíricas. La prueba de hipótesis se puede utilizar para demostrar que la emergencia de ciertos comportamientos en el sistema cumple ciertas condiciones, al igual que permite examinar la viabilidad de los modelos. La explicación que ofrece la simulación se orienta a facilitar la comprensión del porqué se producen ciertos comportamientos en el sistema; en este caso, la simulación parte de identificar los elementos que subyacen a los procesos estudiados y postula posibles consecuencias a partir de la observación de los resultados de la simulación. Si los resultados observados son coherentes con los comportamientos del sistema real, entonces la simulación está proporcionando una explicación plausible.

La simulación basada en agentes puede también ser empleada en procesos de formación, educación y entretenimiento. Para la formación, la ABMs puede proporcionar una representación interactiva, dinámica y lo suficientemente completa en información de un entorno determinado; por ejemplo, los simuladores de vuelo o los simuladores para toma de decisiones, entre otros. Con propósitos educativos, la simulación puede permitir que el usuario experimente en la realidad creada, cambiando las reglas de determinadas variables, los rasgos de los agentes, entre otros aspectos que le permiten identificar por sí mismo las relaciones y principios del sistema. Finalmente, la simulación permite el entretenimiento porque se trata de construcciones de realidades virtuales [30].

La importancia de la modelación y simulación basada en agentes para el estudio de sistemas complejos, ha estimulado el desarrollo de una amplia variedad de software especializado (véase la tabla 3), cuya elección depende principalmente de las preferencias del investigador. La ABMS no es un campo de investigación homogéneo o intelectualmente unificado $[68,69]$. La multiplicidad de escalas que puede abordar (átomos, moléculas, personas, organizaciones, etc.), la inter/trans/multidisciplinariedad que 
la caracteriza (ingeniería, biología, administración, economía, entre otras), y la pluralidad de objetivos a lograr (predicción, prueba de hipótesis, explicación), hacen que la ABMS no sea integrable en un marco de investigación que reúna todas las líneas. Lo único que existe en común entre los investigadores que se soportan en esta técnica son los elementos de la modelación (agentes, interacciones, entorno), y el interés por comprender sistemas que exhiben comportamientos propios emergentes.

Tabla 3. Herramientas computacionales utilizadas para el desarrollo de ABMS

\begin{tabular}{|c|c|c|}
\hline Nombre & Descripción & Licencia \\
\hline \multirow{2}{*}{$\begin{array}{c}\text { Mason } \\
{[70]}\end{array}$} & Trabaja complejidad social mediante modelado físico y modelado abstracto. & \multirow{2}{*}{$\begin{array}{l}\text { Licencia libre académica } \\
\text { (código abierto) }\end{array}$} \\
\hline & Permite el aprendizaje automático. & \\
\hline \multirow{2}{*}{$\begin{array}{c}\text { Swarm } \\
{[71]}\end{array}$} & Requiere conocimiento avanzado en la programación orientada a objetos. & \multirow{2}{*}{$\begin{array}{l}\text { Licencia libre disponible } \\
\text { GPL }\end{array}$} \\
\hline & Orientada al paradigma "framework and lybrary". & \\
\hline $\begin{array}{c}\text { Repast } \\
{[72]}\end{array}$ & Permite el estudio de ciencias sociales. & $\begin{array}{l}\text { Licencia libre disponible } \\
\text { BSD }\end{array}$ \\
\hline \multirow{5}{*}{$\begin{array}{l}\text { NetLogo } \\
{[73]}\end{array}$} & Diseñado para ser usado por primera vez de forma rápida. & \multirow{5}{*}{$\begin{array}{l}\text { Libre con algunas restric- } \\
\text { ciones de modificación, } \\
\text { sin código abierto. }\end{array}$} \\
\hline & Interfaz gráfica amigable con el usuario. & \\
\hline & Lenguaje de fácil entendimiento. & \\
\hline & Permite grabar videos de los modelos. & \\
\hline & $\begin{array}{l}\text { Permite exportar datos a otros programas para posterior tratamiento y aná- } \\
\text { lisis. }\end{array}$ & \\
\hline \multirow{3}{*}{$\begin{array}{l}\text { ORIS } \\
{[74]}\end{array}$} & Utilizado para la enseñanza. & \multirow{3}{*}{$\begin{array}{l}\text { Propiedad libre para las } \\
\text { instituciones académicas }\end{array}$} \\
\hline & Permite la programación por objetos concurrentes. & \\
\hline & Maneja sistemas multiagente. & \\
\hline $\begin{array}{l}\text { AnyLogic } \\
{[75]}\end{array}$ & Permite el estudio de ciencias sociales y naturales. & Solo para propietario \\
\hline $\begin{array}{l}\text { StarLogo } \\
\quad[76]\end{array}$ & $\begin{array}{l}\text { Permite a estudiantes modelar el comportamiento de sistemas descentraliza- } \\
\text { dos, usuario amigable para estudiantes de primaria y educación secundaria. }\end{array}$ & $\begin{array}{l}\text { Libre (código cerrado) } \\
\text { Clearthought Software } \\
\text { License, Version } 1.0\end{array}$ \\
\hline
\end{tabular}

Fuente: elaboración propia

Con el fin de ilustrar algunas de las características de la modelación y simulación basada en agentes, se presenta la simulación desarrollada por Posada [77] en NetLogo, como ejemplo base de la actual investigación, la cual busca comparar la agilidad para aprovechar las oportunidades del entorno y hacer frente a las amenazas que se presentan entre una organización empresarial organizada de manera jerárquica y una organización empresarial que actúa de manera autoorganizada; es decir, en ausencia de un líder/estratega central que determine el comportamiento del sistema. En la simulación, la agilidad se entiende como la oportunidad para hacer frente a los cambios del entorno, y se mide a través del número de actuaciones conjuntas que los individuos desarrollan y permiten aprovechar las oportunidades y tratar las amenazas. Los individuos están organizados en equipos por proceso dentro de cada organización, lo que define las personas con las que interactúan al inicio de la simulación. En la medida en la que avanza el juego, el individuo puede ir estableciendo relaciones con otras personas de la organización.

El modelo de simulación consta de tres partes básicas. En la primera, los individuos de cada organización detectan las oportunidades y/o amenazas, e informan a sus compañeros de equipo de la existencia de estas, a fin de tratarlas a través del desarrollo 
de propuestas de acción. Las oportunidades tienen un tiempo de vida, es decir, pueden desaparecer sin ser aprovechadas. En la segunda, se reconoce que no todos los individuos son susceptibles de aceptar la invitación a desarrollar propuestas de acción. Se valora la susceptibilidad a partir de la combinación de tres variables intrínsecas al individuo: (a) las creencias de la persona respecto al beneficio que el desarrollo de la propuesta de acción le puede generar a él y a la organización en su conjunto; (b) la confianza que tiene la persona que recibe la invitación sobre el que la emitió; y (c) la relación de poder (influencia) que existe entre quien emite la invitación y quien la recibe. Es importante señalar que la variable poder permite distinguir el comportamiento de la organización jerárquica con respecto a la autoorganizada. En la organización jerárquica tienen mayor probabilidad de consolidarse en actuación conjunta aquellas que son propuestas por individuos con poder (mejor posición jerárquica); esto es, la variable poder tiene mayor relevancia en la emergencia de actuaciones conjuntas en la organización jerárquica. En la organización autoorganizada las tres variables (creencias, confianza y poder) tienen la misma relevancia. En la tercera parte, se visualiza la emergencia de actuaciones conjuntas en las dos organizaciones para afrontar oportunidades y/o amenazas. La simulación permite evidenciar que el sistema autoorganizado presenta más cantidad de actuaciones conjuntas que le permiten aprovechar mayor cantidad de oportunidades y tratar más amenazas.

En la figura 2 se presenta la forma en que se visualiza el avance de la simulación. Los círculos color morado representan las organizaciones, las fechas, las oportunidades y amenazas, las manchas blancas representan la información que emite una persona cuando invita a sus contactos a desarrollar una propuesta de acción para tratar oportunidades y/o amenazas, y los círculos azul claro y azul oscuro son los mercados disponibles para las dos organizaciones. La simulación permite llevar un monitoreo gráfico del comportamiento, las actuaciones y los resultados alcanzados por cada organización. En las gráficas, las líneas claras (OJ) representan a la organización jerárquica, y las oscuras (AO) a la autoorganizada.

Por último, es importante reconocer que si bien la ABMs tiene múltiples ventajas, también tiene importantes restricciones. Una de las más relevantes es la dificultad de validación. De hecho, el principal enfoque para tal fin es la verificación interna, la cual consiste en garantizar que los comportamientos que exhibe el sistema no son predefinidos por la programación (errores de código o

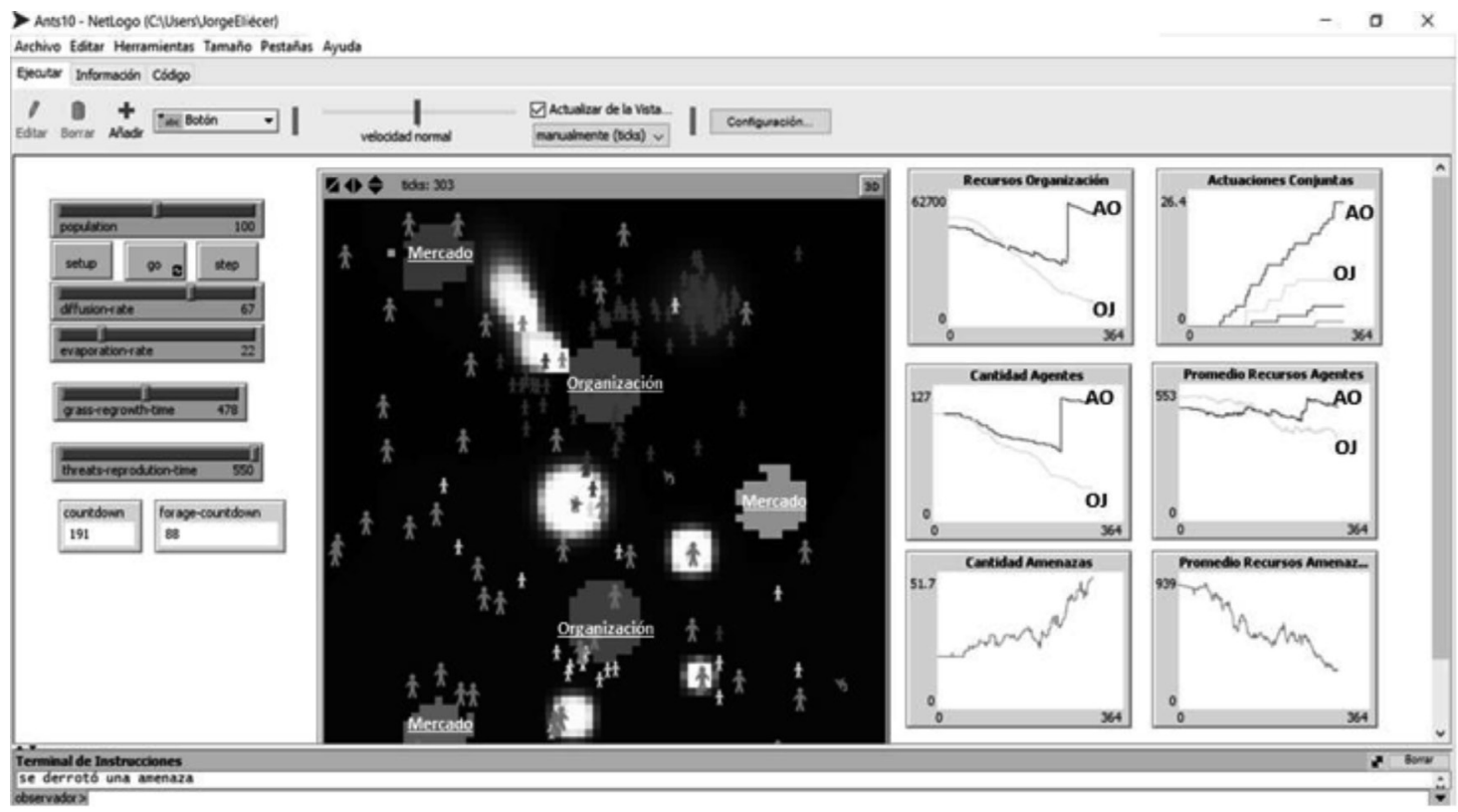

Figura 2. Simulación de la organización jerárquica vs. organización autoorganizada Fuente: [77] 
manipulación por parte del programador), sino que realmente son situaciones emergentes [30]. En la tabla 4 , se presentan otras restricciones, tanto de la ABMS, como de los enfoques tradicionales.

Tabla 4. Desventajas entre modelado basado en agentes y modelos tradicionales

\begin{tabular}{|c|c|}
\hline Modelado basado en agentes & $\begin{array}{c}\text { Modelos tradicionales soportados en deducción y/o } \\
\text { inducción }\end{array}$ \\
\hline $\begin{array}{l}\text { - Verificación de código computacional para que se } \\
\text { ajuste a las especificaciones del modelo } \\
\text { - Difícil validación } \\
\text { - Descripción incompleta del modelo que dificulta la } \\
\text { usabilidad y extensibilidad de los resultados } \\
\text { - Necesidad de gran cantidad de información para una } \\
\text { simulación aproximada a la realidad } \\
\text { - Renuncia a la robustez y generalización de hallazgos } \\
\text { por uso de heterogeneidad y de atributos individuales }\end{array}$ & $\begin{array}{l}\text { - Número limitado de observaciones en el tiempo } \\
\text { - Tamaños pequeños de muestra } \\
\text { - Variables no controladas que reducen la validez y ge- } \\
\text { neralización de los resultados } \\
\text { - Reducción de comportamientos agregados emer- } \\
\text { gentes a estructuras causales con retroalimentación, } \\
\text { ignorando la heterogeneidad de los individuos, asu- } \\
\text { miendo que se mezclan de forma perfecta y son ho- } \\
\text { mogéneos entre sí } \\
\text { - Uso de ecuaciones diferenciales deterministas en el } \\
\text { manejo de fuentes aleatorias } \\
\text { - Generación de informes sobre la estructura del siste- } \\
\text { ma y las políticas que guían las decisiones }\end{array}$ \\
\hline
\end{tabular}

Fuente: elaboración propia

\section{Discusión y conclusiones}

Las técnicas de modelación y simulación en la investigación en administración e ingeniería se han soportado principalmente en el uso de la estadística y la matemática clásica. Este enfoque es pertinente cuando el sistema observado no cambia o lo realiza lentamente. Cuando el objeto de estudio se caracteriza por la complejidad creciente, es decir, por la presencia de comportamientos emergentes a partir de la interacción entre las partes, se requiere del uso de técnicas de modelación y simulación que permitan capturar y exhibir mayores niveles de complejidad; por tanto, que faciliten comprender y explorar la dinámica del sistema. Una alternativa para tal fin se encuentra en la modelación y simulación de sistemas complejos (MSSC), cuyo principal sustento se encuentra en las ciencias de la complejidad. La técnica de Mssc de mayor aplicación en el estudio de sistemas sociales ha sido la modelación y simulación basada en agentes (ABMS).

En la investigación en administración, la ABMS proporciona una amplia variedad de beneficios en el desarrollo de teoría y en la orientación del trabajo empírico. Esta herramienta facilita identificar, explorar y comprender los rasgos que subyacen a los comportamientos emergentes que exhiben los sistemas complejos, como lo son las organizaciones empresariales, y, a partir de esto, formular hipótesis que examinen la pertinencia de planteamientos teóricos y faciliten validar, en el mundo real o en un mundo simulado, las explicaciones generadas.

La investigación en administración y la ingeniería no solo se puede beneficiar con las ventajas que ofrece la MSsc, y puntualmente con las bondades de la ABMs, sino que adicionalmente puede aportar en la construcción de lineamientos que permitan una mayor apropiación de esta técnica en la investigación en las ciencias sociales. La MSsC ha tenido sus mayores avances en la matemática y las ciencias de la computación, y ha sido aplicada, principalmente, al estudio de sistemas naturales y sistemas artificiales. Sin embargo, son los sistemas sociales humanos los que exhiben los mayores niveles de complejidad y, en consecuencia, los que más podrían aportar en el fortalecimiento de esta herramienta.

Si se reconocen los altos niveles de complejidad que exhiben los sistemas sociales humanos, se puedeapreciar el potencial del aporte que disciplinas 
como la administración le pueden hacer al estudio de los sistemas complejos, y puntualmente a las técnicas para la modelación y simulación. El aporte de la administración podría permitir, entre otras cosas, dejar de lado el uso de expresiones metafóricas para referirse a los rasgos que subyacen a la complejidad de los sistemas humanos, y que usualmente han llegado de la matemática, la física y la química, a través de expresiones como "organizaciones como sistemas no lineales", "estructuras disipativas", o "redes autocatalíticas", entre otras [78-79].

Los beneficios y aportes que la investigación en administración puede recibir y/o hacer a través del uso de la MSsc generan importantes retos que exigen, entre otras cosas: (a) acercar los estudiantes al uso de técnicas como modelación y simulación basada en agentes; (b) reconocer que la formación disciplinaria se hace cada vez más difusa y, en consecuencia, el conocimiento no se encuentra parcelado; y (c) promover la socialización completa de los resultados de la investigación.

Acercar los estudiantes al uso de técnicas como modelación y simulación basada en agentes puede implicar modificar los planes de estudio, y de manera amplia, modificar el currículo de tal forma que permita al estudiante reconocer cuándo el uso de la MSsC es pertinente, así como definir los criterios y requisitos para el diseño de la simulación, e interpretar los resultados obtenidos.

El reconocimiento del carácter difuso de la formación disciplinaria, sin lugar a dudas pone de manifiesto el rechazo al reduccionismo que la ha caracterizado. Usualmente, a los estudiantes se les ofrecen cursos prolijamente "empaquetados", en los que cada ciencia tiene su propia parcela y existe una modesta superposición entre ellas [80]. Sin embargo, el avance del conocimiento ha generado un sorprendente fenómeno de convergencia que lleva al surgimiento de nuevas materias, profesiones e investigaciones altamente interdisciplinarias, de acuerdo con Gell-Man (1984), citado por Pagels [80].

El progreso dela ciencia exige del trabajo en comunidades inter/trans/multidisciplinarias. Y los fenómenos complejos requieren de más de un método para investigarlos adecuadamente [81]. El uso de diferentes metodologías, métodos, y técnicas en combinación, puede generar más beneficios que los obtenidos por una sola aproximación [82].
El tercer reto que implica el uso de la Mssc en la investigación en administración exige compartir abiertamente el conocimiento generado, de tal forma que se facilite la usabilidad y extensibilidad de los resultados. La usabilidad se orienta a que todo aquel que corra el programa pueda interpretar las salidas, y entender cómo trabaja la simulación. Esto permite, si se requiere, comparar los resultados de distintas simulaciones que observan el mismo fenómeno. La extensibilidad busca que la simulación pueda ser empleada como punto de partida de nuevas investigaciones, o pueda adaptarse (incluso reescribiendo líneas de código) para otros usos [19]. Compartir los resultados de la investigación incluye describir detalladamente la simulación (qué hace, qué puede hacer, qué variaciones podría tener, etc.), así como brindar plataformas en las que los usuarios puedan acceder al código sin ninguna restricción.

A la fecha, un amplio porcentaje de resultados de modelación y simulación de sistemas complejos realizados desde la matemática, las ciencias de la computación, las ciencias de la tierra y la biología, se encuentran disponibles (véase, por ejemplo, https://ccl.northwestern.edu/netlogo/; o http:// www.dmason.org/). Este hecho no solo evidencia el carácter colaborativo de las investigaciones que se soportan en MSsC, sino que además brinda importantes puntos de partida para la investigación en administración.

La simulación desarrollada por Posada [77] y descrita en el apartado anterior, fue construida a partir de los desarrollos de tres simulaciones previas: el modelo de colonia de hormigas [83], el modelo depredador-presa [84], y el modelo Packet World [85]. El uso de la técnica de ABMs para el estudio de sistemas complejos como lo son las organizaciones empresariales, usualmente no parte de cero; puede partir de la identificación de aspectos en común con otros sistemas complejos ya modelados. En este caso, la comunicación directa e indirecta entre los agentes, la presencia de amenazas y el desarrollo de acciones sincronizadas entre dos o más agentes, respectivamente.

En fin, es necesario reconocer que la ABMs ofrece importantes ventajas para la investigación en sistemas sociales, sin embargo, también tiene restricciones que exigen a los investigadores abordar los problemas a partir del uso de técnicas complementarias. Una importante tendencia en la investigación en 
sistemas complejos es la construcción de paradigmas híbridos que combinen diferentes técnicas (modelación y simulación de agentes, autómatas celulares, e incluso dinámica de sistemas) que permitan aprovechar potencialidades y superar restricciones.

Para próximas investigaciones se sugiere implementar este estudio en diversos tipos de organizaciones de acuerdo con su tamaño y objeto social, comparando y determinando las mejores técnicas de modelación y simulación, de modo que se aprovechen mejor los atributos de los agentes que componen el sistema.

\section{Referencias}

[1] P. Allen, S. Maguire \& B. McKelvey, The Sage Handbook of Complexity and Management, Sage Publications Ltd., Londres, 2011.

[2] E. Mitleton-Kelly, Complex systems and evolutionary perspectives on organisations. The application of complexity theory to organisations, London School of Economics, Londres, 2003.

[3] J. R. Harrison, L. Zhiang, G. R. Carroll \& K. M. Carley, "Simulation Modeling in Organizational and Management Research", Academy Of Management Review, vol. 32, n. ${ }^{\circ}$ 4, pp. 1229-1245, 2007. Disponible en: doi: 10.5465/AMR.2007.26586485

[4] C. Gershenson, "The Implications of Interactions for Science and Philosophy", Foundations of Science, vol. $18, \mathrm{n}^{\circ} 4$, pp. 781-790, 2011. doi: http://dx.doi. org/10.1007/s10699-012-9305-8

[5] C. Maldonado \& N. Gómez, El mundo de las ciencias de la complejidad. Una investigación sobre qué son, su desarrollo y posibilidades, Bogotá, Universidad del Rosario, 2011, pp. 48-74. doi: http://dx.doi. org/10.13140/RG.2.1.4543.5286

[6] J. R. Harrison \& G. R. Carroll, Culture and demography in organizations, Princeton, Princeton University Press, 2006, pp. 166-167.

[7] L. E. Bohorquez \& J. Posada, "Design of a Model of Emerging Joint Actions in Human Social Systems based on the Interaction between Agents", en Workshop on Engineering Applications: International Congress on Engineering (WEA), Bogotá, oct. 2830, 2015, pp. 1-6. doi: http://dx.doi.org/10.1109/ WEA.2015.7370140

[8] N. Siggelkow \& D. Levinthal, “Temporarily Divide to Conquer: Centralized, Decentralized, and Reintegrated Organizational Approaches to Exploration and Adaptation", Organization Science, vol. 14, n. ${ }^{\circ}$
6, pp. 650-669, 2003. doi: http://dx.doi.org/10.1287/ orsc.14.6.650.24870

[9] B. Kogut, J. Colomer \& M. Belinky, "Structural equality at the top of the corporation: Mandated quotas for women directors", Strategic Management Journal, vol. 35 , n 6, pp. 891-902, 2014. doi: http:// dx.doi.org/10.1002/smj.2123

[10] N. Siggelkow \& J. Rivkin, "Speed and Search: Designing Organizations for Turbulence and Complexity", Organization Science, vol. 16, $\mathrm{n}^{\circ}$ 2, pp. 101-122, 2005. doi: http://dx.doi.org/10.1287/orsc.1050.0116

[11] A. Whitehead, Science and the Modern World View, Cambridge, Cambridge University Press, 1925. doi: http://dx.doi.org/10.1063/1.3060085

[12] W. S. McCulloch \& W. Pitts, "A logical calculus of the ideas immanent in nervous activity", Bulletin of mathematical biophysics, vol. 5, n. ${ }^{\circ}$ 4, pp. 115-133, 1943. doi: http://dx.doi.org/10.1007/BF02478259

[13] N. Wiener, Cybernetics: Or, Control and Communication in the Animal and the Machine, Cambridge, MIT Press, 1961.

[14] L. von Bertalanffy, General Systems Theory: Foundations, Development, Applications, New York, George Braziller, 1968.

[15] J. Pollack, D. Adler \& S. Sankaran, "Mapping the field of complexity theory: A computational approach to understanding changes in the field", Emergence: Complexity and Organization, vol. 16, n. ${ }^{\circ} 2$, pp. 74 92, 2014.

[16] L. E. Bohórquez \& A. Espinosa, “Theoretical approaches to managing complexity in organizations: A comparative analysis", Estudios Gerenciales, vol. 31, n. ${ }^{\circ}$ 134, pp. 20-29, 2015. doi: http://dx.doi. org/10.1016/j.estger.2014.10.001

[17] S. Beer, Brain of the firm, New York, John Wiley \& Sons, 1981.

[18] S. Beer, The heart of enterprise, New York, John Wiley \& Sons, 1988.

[19] R. Axelrod, The complexity of cooperation: Agent-based models of competition and collaboration, Princeton, Princeton University Press, 1997. doi: http://dx.doi. org/10.1002/(SICI)1099-0526(199801/02)3:3<46::AIDCPLX6>3.0.CO;2-K

[20] B. Lichtenstein \& B. McKelvey, "Complexity science and computational models of emergent order: What's there? What's missing?", en Academy of Management Annual Meeting, New Orleans, LA, 2004.

[21] S. Kauffman, Investigations, Oxford, Oxford University Press, 2000.

[22] S. Maguire, B. McKelvey, M. Laurent \& N. Ôztas, "Complexity science and organization studies", en The Sage Handbook of Organization Studies, S. Clegg, 
C. Hardy, T. Lawrence y N. Walter, Eds., Londres, Sage Publications, 2006, pp. 165-214. doi: http://dx.doi.org/10.4135/9781848608030.n6

[23] D. Helbing, "Globally networked risks and how to respond", Nature, vol. 497, n. ${ }^{\circ}$ 7447, pp. 51-59, 2014. doi: http://dx.doi.org/10.1038/nature12047

[24] D. J. Watts, Seis grados de separación: la ciencia de las redes en la era de acceso, Barcelona, Paidós, 2006.

[25] I. Prigogine \& I. Stengers, La nueva alianza: Metamorfosis de la ciencia, Madrid, Alianza Editorial, 2002.

[26] C. Emmeche, Vida simulada en el ordenador, Barcelona, Gedisa Editorial, 1998.

[27] D. M. Kreps, A course in microeconomic theory, Princeton, NJ, Princeton University Press, 1990. doi: http://dx.doi.org/10.1002/mde.4090130112

[28] N. Gilbert \& K. Troitzsch, Simulación en las Ciencias Sociales, 2a. edición, España, McGraw-Hill, 2006.

[29] R. Axelrod \& M. Cohen, Harnessing Complexity, EE.UU., Basic Books, 2000.

[30] R. Axelrod, "Advancing the Art of Simulation in the Social Sciences", en Handbook of Research on Nature Inspired Computing for Economy and Management, J.P. Hershey, Ed., EE.UU.: Idea Group, 2005, p. 90100. doi: http://dx.doi.org/10.1002/(SICI)10990526(199711/12)3:2<16::AID-CPLX4>3.0.CO;2-K

[31] R. Axtell, "Why agents?: on the varied motivations for agent computing in the social sciences", en Workshop on Agent Simulation: Applications, Models, and Tools, C. Macal y D. Sallach, Eds., Chicago, The University of Chicago, 2000, pp. 3-24. doi: http://dx.doi. org/10.1002/(SICI)1099-0526(199711/12)3:2<16:AIDCPLX4>3.0.CO;2-K

[32] R. M. Burton, "Computational laboratories for organization science: Questions, validity and docking”, Computational \& Mathematical Organization Theory, vol. 9, n. ${ }^{\circ}$ 2, pp. 91-108, 2003. doi: http://dx.doi. org/10.1023/B:CMOT.0000022750.46976.3c

[33] K. J. Cohen \& R. M. Cyert, "Simulation of organizational behavior", en Handbook of organizations, J. G. March, Ed., Chicago, Rand McNally, 1965, pp. 305-334.

[34] M. W. Macy \& R. Willer, "From factors to actors: Computational sociology and agent-based modeling", Annual review of sociology, vol. 28, pp. 143166, 2002. doi: http://dx.doi.org/10.1146/annurev. soc.28.110601.141117

[35] J. M. Epstein \& R. Axtell, Growing artificial societies: Social science from the bottom up, Washington, DC,: Brookings Institution, 1996.

[36] K. Kim \& S. Cho, "A comprehensive overview of the applications of artificial life", Artificial Life, vol. 12, n. ${ }^{\circ}$ 1, pp. 153-182, 2006. doi: http://dx.doi. org/10.1162/106454606775186455

[37] C. M. Macal \& M. J. North, "Tutorial on agent-based modelling and simulation", Journal of simulation, vol. 4, n. ${ }^{\circ}$ 3, pp. 151-162, 2010. doi: http://dx.doi. org/10.1057/jos.2010.3

[38] M. D. Cohen, J. G. March \& J. P. Olsen, "A garbage can model of organizational choice", Administrative Science Quarterly, vol. 17, pp. 1-25, 1972. doi: http:// dx.doi.org/10.2307/2392088

[39] R. M. Cyert \& J. G. March, "A behavioral theory of the firm", en Organizational Behavior 2: Essential Theories of Process and Structure, J. Miner, Ed., Armonk, M.E. Sharpe, 1963, pp. 60-77.

[40] R. M. Burton \& B. Obel, "A computer simulation test of the M-form hypothesis", Administrative Science Quarterly, vol. 25, pp. 457-466, 1980. doi: http://dx.doi.org/10.2307/2392263

[41] T. W. Malone, "Modeling coordination in organizations and markets", Management Science, vol. 33, n. ${ }^{\circ} 10$, pp. 1317-1332, 1986. doi: http://dx.doi. org/10.1287/mnsc.33.10.1317

[42] M. Masuch \& P. LaPotin, "Beyond garbage cans: An AI model of organizational choice”, Administrative Science Quarterly, vol. 34, pp. 38-67, 1989. doi: http://dx.doi.org/10.2307/2392985

[43] J. F. Padgett, "Managing garbage can hierarchies", Administrative Science Quarterly, vol. 25, pp. 583-604, 1980. doi: http://dx.doi.org/10.2307/2392282

[44] J. L. G. V. Medina, "Explicación, mecanismo y simulación: otra manera de hacer sociología", Empiria: Revista de metodología de ciencias sociales, n. ${ }^{\circ} 28$, pp. 35-58, 2014. doi: http://dx.doi.org/10.5944/empiria.28.2014.12120

[45] N. Gilbert, "Simulation as a Research Strategy", en Social Science Microsimulation, K. Troitzsch, U. Mueller, N. Gilbert \& J. Doran, Eds., Berlín, Springer, 1996, pp. 448-454.

[46] W. S. Kim, "Effects of a trust mechanism on complex adaptive supply networks: An agent-based social simulation study", Journal of Artificial Societies and Social Simulation, vol. 12, n. ${ }^{\circ}$ 3, pp. 1-4, 2009. Disponible en: http://jasss.soc.surrey.ac.uk/12/3/4.html

[47] E. Bonabeau, "Agent-based modeling: Methods and techniques for simulating human systems", en Proceedings of the National Academy of Sciences, vol. 99, suppl. 3, pp. 7280-7287, 2002. doi: http://dx.doi. org/10.1073/pnas.082080899

[48] N. Gilbert, "Computational social science: Agentbased social simulation", Centre for Research on Social Simulation, 2007. Disponible en: http://epubs. surrey.ac.uk/1610/1/fulltext.pdf 
[49] C. Nikolai \& G. Madey, “Tools of the trade: A survey of various agent based modeling platforms", Journal of Artificial Societies and Social Simulation, vol. 12, n. ${ }^{\circ}$ 2, pp. 1-2, 2009. Disponible en: http://jasss.soc. surrey.ac.uk/12/2/2.html

[50] N. Gilbert \& K. Troitzsch, Simulation for the social scientist, Berkshire, McGraw-Hill Education, 2005, pp. 172-197. Disponible en: http://www.modares. ac.ir/uploads/Agr.Oth.Lib.16.pdf

[51] C. Sansores \& J. Pavón, "Simulación social basada en agentes. Inteligencia artificial", Revista Iberoamericana de Inteligencia Artificial, vol. 9 n. ${ }^{\circ}$ 25, pp. 71-78, 2005. Disponible en: http://oai.redalyc.org/articulo. oa?id=92592509

[52] C. Macal \& M. J. North, "Agent-based modeling and simulation", en Winter simulation conference, Orlando, FL, IEEE, 2009, pp. 86-98. doi: http://dx.doi. org/10.1109/WSC.2009.5429318

[53] M. J. North \& C. M. Macal, Managing business complexity: discovering strategic solutions with agentbased modeling and simulation, Oxford, Oxford University Press, 2007.

[54] S. Bandini, S. Manzoni \& G. Vizzari, "Agent based modeling and simulation: an informatics perspective", Journal of Artificial Societies and Social Simulation, vol. 12, n. ${ }^{\circ}$, pp. 1-4, 2009. Disponible en: http://jasss.soc.surrey.ac.uk/12/4/4.html

[55] G. Weisbuch \& S. T. Ryckebusch, Complex systems dynamics: An introduction to automata networks. Reading, EE.UU., Addison-Wesley/ Addison Wesley Longman, 1991. doi: http://dx.doi. org/10.1063/1.4822990

[56] J. H. Holland, Hidden Order: How Adaptation Builds Complexity, Ee.uU., Perseus Books, 1995.

[57] C. G. Langton, Artificial life: An Overview, Redwood City, CA, Addison-Wesley Publishing Company, 1989. doi: 10.1002/(SICI)1099-1743(199701/02)14: $1<78::$ AID-SRES139>3.0.CO;2-0

[58] G. D. M. Serugendo, M. P. Gleizes \& A. Karageorgos, "Self- organisation and emergence in MAs: An overview”, Informatica, vol. 30, n. ${ }^{\circ}$ 1, pp. 45-54, 2006.

[59] W. B. Arthur, S. N. Durlauf \& D. A. Lane (Eds.), The Economy as an Evolving Complex System II, SFI Studies in the Sciences of Complexity, Reading, EE.UU., Addison-Wesley, 1997.

[60] M. J. North, C. M. Macal, J. S. Aubin, P. Thimmapuram, M. Bragen, J. Hahn et al., "Multiscale agent-based consumer market modeling", Complexity, vol. 15, n. ${ }^{\circ}$ 5, pp. 37-47, 2010. doi: http://dx.doi.org/10.1002/ cplx.20304

[61] C. M. Macal, "Emergent structures from trust relationships in supply chains", en Proceedings of Agent 2004: Conference on Social Dynamics: Interaction,
Reflexivity and Emergence, C. Macal, D. Sallach \& M. North, Eds., Chicago, Argonne National Laboratory, 2004, pp. 743-760.

[62] L. Yin, "Assessing Indirect Spatial Effects of Mountain Tourism Development: an Application of Agent-based Spatial Modeling", Tourism and Regional Science, vol. 37, n. ${ }^{\circ}$ 3, pp. 257-265, 2007.

[63] A. Chandrasekaran, K. Linderman, F. J. Sting \& M J. Benner, "Managing R\&D Project Shifts in HighTech Organizations: A Multi-Method Study", Production and Operations Management, vol. 25, n. ${ }^{\circ}$ 3, pp. 390-416, 2016. doi: http://dx.doi.org/10.1111/ poms. 12410

[64] N. Altay \& R. Pal, "Information diffusion among agents: implications for humanitarian operations", Production and Operations Management, vol. 23, n. ${ }^{\circ}$ 6, pp. 1015-1027, 2014. doi: http://dx.doi. org/10.1111/poms.12102

[65] K. D. Miller, B. T. Pentland \& S. Choi, "Dynamics of performing and remembering organizational routines", Journal of Management Studies, vol. 49, n. ${ }^{\circ}$, pp. 1536-1558, 2012. doi: http://dx.doi.org/10.1111/ j.1467-6486.2012.01062.x

[66] P. L. Bylund, “Signifying Williamson's Contribution to the Transaction Cost Approach: An Agent-Based Simulation of Coasean Transaction Costs and Specialization", Journal of Management Studies, vol. 52, n. ${ }^{\circ}$ 1, pp. 148-174, 2015. doi: http://dx.doi. org/10.1111/joms. 12110

[67] V. A. Aggarwal, N. Siggelkow \& H. Singh, "Governing collaborative activity: interdependence and the impact of coordination and exploration", Strategic Management Journal, vol. 32, n. ${ }^{\circ}$ 7, pp. 705-730, 2011. doi: http://dx.doi.org/

[68] 10.1002/smj.900

[69] M. Wheeler, S. Bullock, E. Di Paolo, J. Noble, M. Bedau \& P. Husbands, "The view from elsewhere: perspectives on alife modelling”, Artificial Life, vol. 8, n. ${ }^{\circ}$ 1, pp. 87-100, 2002. doi: http://dx.doi. org/10.1162/106454602753694783

[70] K. Mainzer, Thinking in complexity: The computational dynamics of matter, mind and making, $5^{\mathrm{a}}$ edición, Berlín, Springer-Verlag, 2007. doi: 10.1007/978-3662-05364-5

[71] Mason [Página de Internet]. Disponible en: http:// cs.gmu.edu/ eclab/projects/ mason/

[72] Swarm [Página de Internet]. Disponible en http:// www.swarm.org/

[73] Repast [Página de Internet]. Disponible en https:// repast.github.io/

[74] Netlogo [Página de Internet]. Disponible en http:// ccl.northwestern.edu/netlogo/ 
[75] ORIs [Página de Internet]. Disponible en http:// www.enib.fr/ harrouet/oris.html

[76] Anylogic [Página de Internet]. Disponible en http:// www.anylogic.com/features/

[77] Starlogo [Página de Internet]. Disponible en http:// education.mit.edu/ portfolio_page/starlogonova/

[78] J. Posada, Diseño de una simulación basada en agentes para la auto-organización empresarial, tesis de Maestría en Ingeniería Industrial, Univ. Distrital Francisco José de Caldas, Bogotá, Colombia, 2016.

[79] W. C.Tsai, "Application of Complexity Science Perspective on New Business Development: A Case Study of visA Organization", Journal of International Management Studies, vol. 9, n. ${ }^{\circ}$ 2, pp. 152-163, 2014.

[80] S. Billinger, N. Stieglitz \& T. R. Schumacher, "Search on rugged landscapes: An experimental study", Organization Science, vol. 25, n. ${ }^{\circ}$ 1, pp. 93-108, 2013. doi: http://dx.doi.org/10.1287/orsc.2013.0829

[81] H. Pagels, Los sueños de la razón: El ordenador y los nuevos horizontes de las ciencias de la complejidad, Barcelona, Gedisa, 1991.

[82] R. Cameron \& S. Sankaran, "Mixed methods research design: Well Beyond the Notion of Triangula- tion", en Novel Approaches to Organizational Project Management Research, N. Drouin, R. Müller \& S. Sankaran, Eds., Copenhague, Copenhagen Business School Press DK, 2013, PP. 383-401.

[83] M. C. Jackson, "Towards coherent pluralism in management science", Journal of the Operational Research Society, vol. 50, no 1, pp. 12-22, 1999. doi: http://dx. doi.org/10.2307/3010384

[84] U. Wilensky, "Netlogo ants model", Center for connected learning and computer-based modeling, Evanston, EE.UU., Northwestern University, 1997. Disponible en: http://ccl. northwestern.edu/netlogo/ models/Ants.

[85] U. Wilensky \& K. Reisman, "Thinking like a wolf, a sheep, or a firefly: Learning biology through constructing and testing computational theories-an embodied modeling approach", Cognition and instruction, vol. 24, n. ${ }^{\circ}$ 2, pp. 171-209, 2006. doi: http:// dx.doi.org/10.1207/s1532690xci2402_1

[86] D. Weyns \& T. Holvoet, "Model for simultaneous actions in situated multi-agent systems", en Multiagent System Technologies, M. Schillo, M. Klusch, J. Müller \& H. Tianfield, Eds., Heidelberg, Springer Berlin Heidelberg, 2003, pp. 105-118. 